\title{
Erben und Vererben
}

G. Hörster-Metzdorf
Wer Vermögen hat, dem ist es oft nicht gleichgültig, was mit diesen Werten nach seinem Tod geschieht. Damit man hier eine bewusste Entscheidung treffen kann, sollte man die Grundzüge der gesetzlichen Erbfolge kennen und - für den Fall, dass man sein Erbe anders verteilen möchte - sich über die Voraussetzungen und Inhalt möglicher Regelungen informieren. Hierbei sollen die nachfolgenden Erläuterungen eine kleine Hilfe sein.

\section{Wer ist Erbe?}

Erbe kann man durch Testament (sog. gewillkürter Erbe) oder, wenn kein Testament vorhanden ist, als gesetzlicher Erbe aufgrund gesetzlicher Erbfolge werden. Die gesetzliche Erbfolge geht davon aus, dass die Verwandten und der Ehepartner des Verstorbenen in ganz bestimmter Reihenfolge als seine Erben eintreten. Zunächst erben die Verwandten der 1. Ordnung - das sind die Kinder und Kindeskinder. Sind Erben der 1.Ordnung nicht vorhanden, sind in der 2 . Erbfolge die Eltern oder deren andere Kinder, also die Geschwister des Verstorbenen, gesetzliche Erben. Sind auch Erben der 2. Erbfolge nicht vorhanden, so kommen in der 3. und 4. Erbfolge die Großeltern oder Urgroßeltern bzw. deren Kindern und Kindeskinder zum Zuge. Gesetzlicher Erbe ist auch der Ehegatte des Verstorbenen. Der Umfang seines Anteils am Nachlass hängt vom Güterstand
(Zugewinngemeinschaft oder Gütertrennung) und von den noch lebenden Angehörigen ab. Mit dem Tod des Erblassers geht der gesamte Nachlass (Vermögen, aber auch Schulden) automatisch in das Eigentum der Erben über.

\section{Was ist ein Testament?}

Das Testament ist eine letztwillige Verfügung des Erblassers, in der er festgelegt hat, was mit seinem Vermögen nach seinem Tod geschehen soll. Hier kann der Erblasser festlegen, wer Erbe sein soll und in welchem Umfang er erben soll bzw. wie die Erben den Nachlass unter sich verteilen sollen (sog. Teilungsanordnung). Er kann einzelne Gegenstände aus seinem Nachlass auch bestimmten Personen zuwenden, ohne dass diese Erben werden (sog. Vermächtnis).

Ein Testament ist nur wirksam, wenn es in der gesetzlich vorgeschriebenen Form errichtet wurde. Es kann notariell oder eigenhändig errichtet werden. Im letzteren Fall muss der gesamte Text handschriftlich vom Erblasser persönlich geschrieben, mit Datum und Ortsangabe versehen und unterschrieben werden.

\section{Was ist ein Pflichtteil?}

Der gesetzliche Pflichtteilsanspruch stellt sicher, dass die Kinder und der Ehegatte bzw. bei Kinderlo- sigkeit auch die Eltern des Erblassers auf jeden Fall eine wirtschaftliche Beteiligung am Nachlass bekommen. Der Pflichtteil beträgt die Hälfte des gesetzlichen Erbteils, also die Hälfte dessen, was diese Angehörigen als gesetzliche Erben bekommen hätten. Die Pflichtteilsberechtigten sind nicht Erben, sondern haben nur einen schuldrechtlichen, auf eine Geldzahlung ausgerichteten Anspruch gegen den Erben.

\section{Was ist ein Vermächtnis?}

Sollen bestimmte Personen nicht Erben werden, sondern nur einzelne Gegenstände aus dem Nachlass erhalten, so kann der Erblasser bezüglich dieser Gegenstände (z.B. ein bestimmtes Schmuckstück oder ein Bild) ein Vermächtnis anordnen. Diese Gegenstände werden nicht mit dem Tod des Erblassers automatisch Eigentum des Bedachten. Er hat lediglich einen Anspruch gegen die Erben auf Herausgabe des zugewandten Gegenstandes.

Was ist eine Teilungsanordnung?

Durch die Teilungsanordnung kann der Erblasser festlegen, wie sein Nachlass unter den Erben verteilt werden soll, z.B. wer bestimmte Immobilien und wer das Barvermögen bekommt. Sind sich allerdings alle Erben einig, dass sie das Erbe anders 